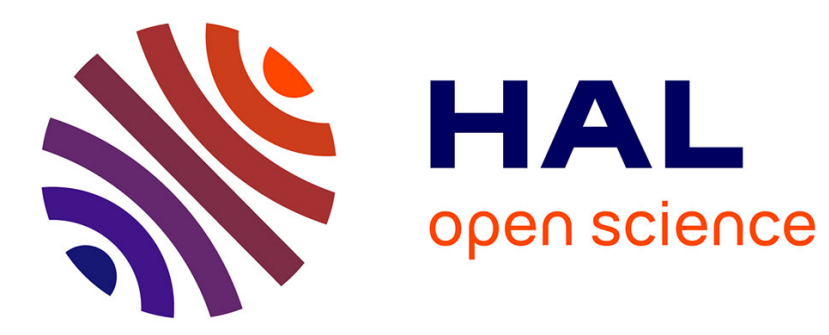

\title{
Quasistatic displacement self-sensing method for cantilevered piezoelectric actuators.
}

Ioan Alexandru Ivan, Micky Rakotondrabe, Philippe Lutz, Nicolas Chaillet

\section{To cite this version:}

Ioan Alexandru Ivan, Micky Rakotondrabe, Philippe Lutz, Nicolas Chaillet. Quasistatic displacement self-sensing method for cantilevered piezoelectric actuators.. Review of Scientific Instruments, 2009, 80 (6), pp.065102-1/065102-8. 10.1063/1.3142486 . hal-00400387

\section{HAL Id: hal-00400387 \\ https://hal.science/hal-00400387}

Submitted on 30 Jun 2009

HAL is a multi-disciplinary open access archive for the deposit and dissemination of scientific research documents, whether they are published or not. The documents may come from teaching and research institutions in France or abroad, or from public or private research centers.
L'archive ouverte pluridisciplinaire HAL, est destinée au dépôt et à la diffusion de documents scientifiques de niveau recherche, publiés ou non, émanant des établissements d'enseignement et de recherche français ou étrangers, des laboratoires publics ou privés. 


\title{
${ }^{1}$ Quasistatic displacement self-sensing method for cantilevered ${ }_{2}$ piezoelectric actuators
}

\author{
3 \\ Ioan Alexandru Ivan, Micky Rakotondrabe, ${ }^{\text {a) }}$ Philippe Lutz, and Nicolas Chaillet \\ Department of Automatic Control and Micro-Mechatronic Systems, FEMTO-ST Institute, \\ UMR CNRS 6174-UFC/ENSMM/UTBM, 24 rue Alain Savary, 25000 Besançon, France
}

(Received 27 March 2009; accepted 4 May 2009; published online xx xx xxxx)

\begin{abstract}
Piezoelectric meso- and microactuator systems required for manipulation or assembly of microscale objects demand reliable force and/or displacement information. Available sensors are prone to dimension restrictions or precision limitation. Self-sensing method, based on the electric charge measurement, may represent a solution in terms of cost-effectiveness and integration, the actuator performing simultaneously as its own sensor. This paper presents a self-sensing method dedicated to free uni- and bimorph piezocantilevers but can also be adapted to other piezoactuator types. The integrated electric current, used to convert the charge, can be compensated against piezoelectric material nonlinearities to provide accurate displacement information. The advantages relative to existing self-sensing methods consist in the ability to keep this displacement information for long-term periods (more than a thousand seconds) and in the reduction in signal noise. After introductive issues related to the method the base principle allowing the estimation of tip displacement is presented. Then, the identification procedure of the estimator parameters is depicted and representative experimental results are shown. Finally, a series of aspects related to electronic circuits are discussed, useful for successful system implementation.
\end{abstract}

\section{I. INTRODUCTION}

23 Piezoelectric cantilevered actuators usually made up of 24 one or two piezoelectric layers (called uni- or bimorph) are 25 present in many micromanipulation and microrobotic appli26 cations, thanks to their high displacement resolution and fast 27 response time. The static and dynamic behaviors of piezo28 electric actuators, their inherent nonlinearities (hysteresis and 29 creep), and limits were studied and modeled ${ }^{1-4}$ especially 30 during the past 2 decades in attempting to provide more ef31 ficient control solutions. ${ }^{5-7}$

32 In order to perform very accurate and fast response time 33 closed-loop micromanipulation tasks, various sensors have 34 been used. Unfortunately, these sensors are not ideally 35 adapted to the micro- and nanoworld because of their sizes, 36 performances, and limited measurement of degrees of free37 dom. Table I summarizes mostly available sensors in the 38 field. Hence, an alternative to the use of sensors is the self39 sensing method. There are several advantages of the self40 sensing method relative to the use of external sensors. 41 Among them, it allows a consistent reduction in the costs by 42 eliminating expensive sensors. As can be seen, resolution can 43 also be submicrometric and comparable to that of external 44 sensors. Self-sensing is based on charge conversion. In fact, 45 charge is nearly proportional to the displacement, hence 46 there is no need to further compensate the complex nonlin47 earities (hysteresis and creep) such as in (Ref. 8).

48 The idea of self-sensing in piezoelectric cantilever has

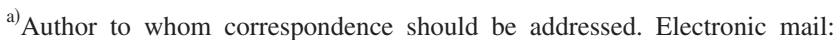
mrakoton@femto-st.fr.
}

been started by the work of Dosch et al. ${ }^{9}$ While it is not a 49 new concept on vibration damping or control, ${ }^{10-13}$ more re- 50 cently, it has shown its feasibility for piezoelectric tubes of $\mathbf{5 1}$ atomic force microscopy. ${ }^{14,15}$ But to our knowledge, self- 52 sensing methods have not yet been adapted for long-term 53 (more than hundreds of seconds) displacement measurement 54 of cantilevered actuators, as required by micromanipulation 55 and microrobotic tasks. In this paper, we present a compen- 56 sated self-sensing approach especially dedicated to long-term 57 static measurement. We especially focus on the displacement $\mathbf{5 8}$ measurement of a piezoelectric cantilever beam.

Drawbacks of displacement self-sensing method refer to 60 inherent charge leaks and temperature influence. With proper 61 actuators and electronic circuits, charge information may be 62 preserved even for thousands of seconds. However, because 63 of the temperature variations, extra care will be required for 64 proper thermal isolation especially in the case of nonsym- 65 metric cantilevers (example: unimorph) to limit temperature- 66 related uncertainties.

There are several self-sensing schematics depending on 68 application. Capacitive bridges ${ }^{9,11}$ are convenient for vibra- 69 tion control but are not easy to balance for long-term mea- 70 surements. Structures with both electrodes for actuation and 71 electrodes for sensing are a simple solution but their incon- 72 venience is a partial reduction in the total actuating 73 range. ${ }^{14,16}$ A current integrator was introduced in Ref. 17 for 74 a piezostack. The disadvantage was a poor compensation of 75 leaking resistance with a very high value potentiometer 76 across the integrating capacitor. Another method quite re- 77 lated to self-sensing concept was linearization of the actuator 78 displacement using voltage-to-charge amplifiers. ${ }^{18,19}$ The ad- 79 
TABLE I. Displacement sensors for the microworld.

\begin{tabular}{|c|c|c|}
\hline Sensor type & Advantages & Disadvantages \\
\hline Triangulation lasers & $\begin{array}{l}\text { High precision and resolution; fair band pass; } \\
\text { and spot measurement }\end{array}$ & $\begin{array}{l}\text { Quite expensive, large sizes, and } \\
\text { limited measurement range }\end{array}$ \\
\hline Interferometers & $\begin{array}{l}\text { Very high precision resolution and range; increased band pass; } \\
\text { and } \operatorname{spot}(\mathrm{s}) \text { measurement }\end{array}$ & Very expensive and large sizes \\
\hline Diffraction grating target & High precision and multidimensional measurement & $\begin{array}{l}\text { Large sizes, require attaching target, } \\
\text { and expensive }\end{array}$ \\
\hline Strain gages & Less expensive and millimeter size & $\begin{array}{l}\text { Fragile, noisy output signal, and } \\
\text { temperature influence }\end{array}$ \\
\hline Capacitive or inductive & High sensibility, high precision, and fair price & $\begin{array}{l}\text { Require linearization, from fair to quite large } \\
\text { dimensions, and close vicinity requirements }\end{array}$ \\
\hline $\begin{array}{l}\text { Magnetic Hall effect, magnetoresistive, } \\
\text { and magnetostrictive }\end{array}$ & Good precision, band pass, and fair price & $\begin{array}{l}\text { Require permanent magnets often too large } \\
\text { and close vicinity requirements. }\end{array}$ \\
\hline Using image processing & Large measurement range and in-plane displacement & $\begin{array}{l}\text { Expensive and limited resolution and } \\
\text { response time }\end{array}$ \\
\hline Piezoelectric self-sensing & $\begin{array}{l}\text { Double functionality, high band pass, high resolution, } \\
\text { and lowest price }\end{array}$ & $\begin{array}{l}\text { Require nonlinear compensation and } \\
\text { long-term charge leaking }\end{array}$ \\
\hline
\end{tabular}

80 vantage was a linear voltage-to-displacement characteristic. 81 However, the conventional HV supplier needs to be replaced 82 by more complex charge driven circuits. In our paper we 83 propose two simple schematics of current integrators (modi84 fied charge amplifiers) that can also be easily implemented 85 onto existing systems, avoiding requiring the redesign of ac86 tuator or HV supply.

87 The developed self-sensing systems can be divided into 88 three main parts, as in Fig. 1: the piezoelectric actuator, the 89 electronic circuit, and the data processing system. The latter 90 is the proposed self-sensing estimator. The estimate displace91 ment could be used for further feedback or closed-loop con92 trol systems.

93 Piezoelectric actuators are submitted to $V_{\text {in }}$ external volt94 ages in a range of up to several hundred volts, depending on 95 actuators. Resulted charge $Q$ (in fact integrated current) is 96 converted by the electronic amplifier to a measurable voltage $97 V_{\text {out }}$. This signal will be converted for further numerical pro98 cessing. Data is further processed on a computer or is de99 ployed into a real-time processor or microcontroller. External 100 signals can be provided to improve the self-sensing accuracy, 101 for instance temperature variation may be compensated with 102 a small thermistor. As the charge cannot be kept indefinitely, 103 external resetting before each measurement prevents satura104 tion and offsets large parts of the static error.

105 Among the contributions of this paper include: the intro106 duction of an antiparallel reference capacitance, numerical 107 compensations of amplifier bias currents and of piezoelectric 108 leaking resistance, and dielectric absorption. A step by step 109 approach of the identification of the self-sensing parameters 110 is presented as well as experimental results.

111 The paper is organized as follows. First, we present the 112 principle and related equations of the self-sensing estimator.

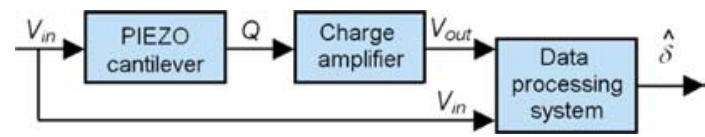

FIG. 1. (Color online) Displacement self-sensing system.
Afterwards, we detail the parameter identification. Hence, we 113 present the experimental results. Finally, we relate some is- 114 sues to be taken into account when deploying self-sensing 115 systems.

\section{DISPLACEMENT DETECTION}

\section{A. Charge output of piezoelectric cantilever}

Consider a bimorph cantilevered beam piezoactuator 119 subjected to an electrical excitation $V_{\text {in }}$ (Fig. 2). The beam is 120 characterized by its length $L$, its width $w$, and its half- 121 thickness $h$.

In the absence of external force, we have a theoretically 123 linear relation between displacement and applied voltage ${ }^{20} \quad 124$

$$
\delta=-\frac{3 d_{31}}{1+\frac{d_{31}^{2}}{4 s_{11}^{E} \varepsilon_{33}^{S}}} \frac{L^{2}}{h^{2}} V_{\text {in }},
$$

where $s_{11}^{E}$ is the compliance coefficient along the beam $(X 126$ direction), $\varepsilon_{33}^{S}$ and $d_{31}$ are dielectric and piezoelectric mate- 127 rial coefficients.

Using the relation between the applied voltage and the 129 capacitance for bimorph piezoelectric cantilever beam

$$
Q=\frac{4 w L \varepsilon_{33}^{S}}{h} V_{\text {in }}
$$

charge directly results and, as stated previously, is quasipro- 132 portional to free displacement $\delta$

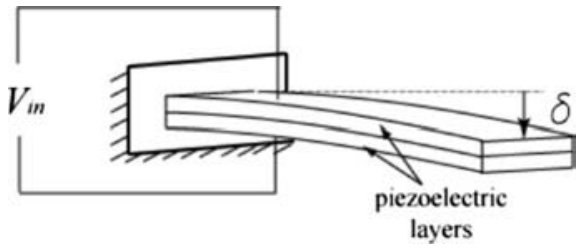

FIG. 2. A bimorph piezoelectric cantilever beam. 


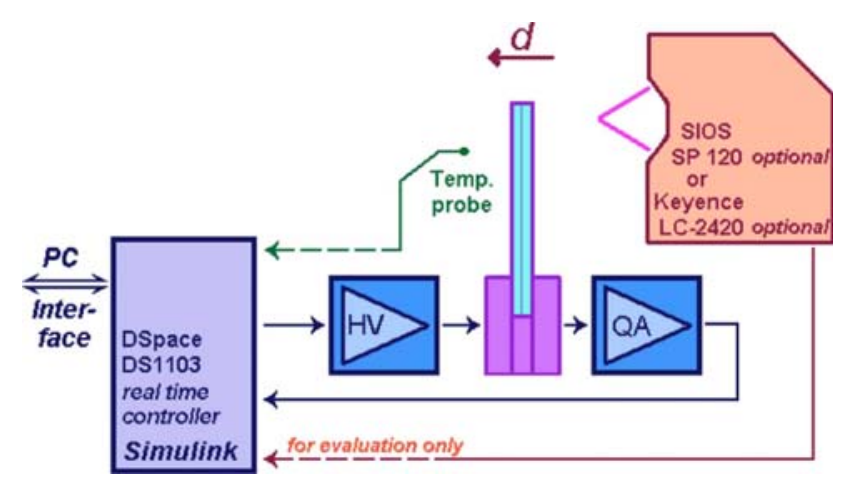

FIG. 3. (Color online) Experimental setup schematic of the self-sensing actuator. Direction for positive displacement is given.

$$
Q=\frac{4 w h \varepsilon_{33}^{S}}{3 d_{31} L}\left(1+\frac{d_{31}^{2}}{4 s_{11}^{E} \varepsilon_{33}^{S}}\right) \delta=\alpha \delta,
$$

135 where $\alpha$ is denoted as an actuator charge-displacement coef136 ficient. In the sequel, this charge will be converted into a 137 measurable voltage $V_{\text {out }}$ from which the deflection $\delta$ will be 138 estimated, as described in Fig. 1.

\section{B. Experimental setup}

140 A schematic overview of the setup is depicted in Fig. 3. 141 Several uni- and bimorph rectangular actuators (PZT on $\mathrm{Cu}$ 142 or Ni substrate) were tested, of length between $10-15 \mathrm{~mm}$, 143 width between 1-2 mm, and total thickness of $0.27-0.45$ $144 \mathrm{~mm}$. A Keyence LC-2420 optical displacement reader was 145 only used for intermediate tests on actuators displacement; 146 for some measurements requiring better precision a SIOS 147 SP-120 miniature plane-mirror interferometer was employed 148 (Fig. 4). However displacement readings served only for ref149 erencing and evaluating purposes of the self-sensing method. 150 The high voltage (HV) amplifier allowed applying a voltage 151 up to $\pm 150 \mathrm{~V}$. A current integrator amplifier circuit (modified 152 charge amplifier) to be discussed next chapter provided $V_{\text {out }}$ 153 output signal. The Matlab Simulink detection model was de154 ployed on a high speed DSpace DS1103 real-time controller 155 board. A PC-based CONTROLDESK interface served for model 156 parameterization and data acquisition/presentation.

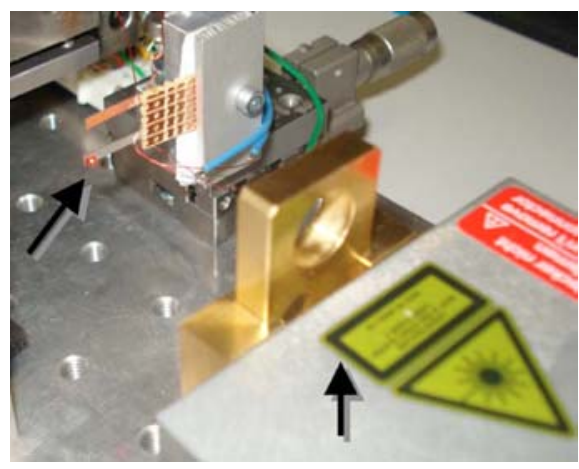

FIG. 4. (Color online) Photo of experimental setup. Actuator is placed in the upper left and SIOS interferometer is in the right side of the image.

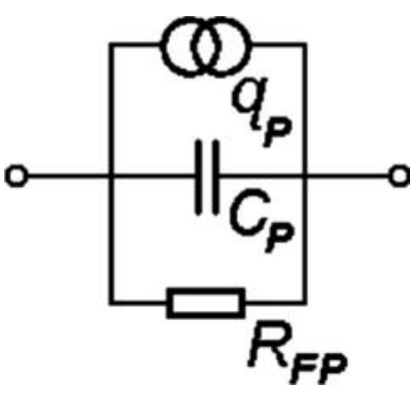

FIG. 5. Equivalent electrical schematic of a piezoactuator.

\section{Integrator amplifier}

The static electrical equivalent schematic of piezoelec- 158 tric bender is a charge generator in parallel with a capacitor 159 and a leaking resistance, as seen in Fig. 5, and its electrome- 160 chanical model is shown in Ref. 2. $C_{P}$ capacitance is in the 161 order of nanofarad depending on the shape and dimensions 162 of the microactuator's structure while $R_{\mathrm{FP}}$ is the insulating 163 resistance, whose order of magnitude is between 164 $10^{9} \ldots 10^{12} \Omega$.

If we ignore nonlinear effects, charge is proportional to 166 the applied voltage and the external force. To measure 167 charge, we propose a precise integrator circuit scheme, as 168 pictured in Fig. 6 and described below.

The input signal $V_{\text {in }}$ is inverted and applied to a "refer- 170 ence capacitor" $C_{R}$ whose the value is close to $C_{P}$ value; it 171 will "absorb" a significant part of the charge due to the ap- 172 plied voltage, according to the second Kirchoff law. Al- 173 though $C_{R}$ and $\mathrm{HV}$ inverter may miss from the circuit, their 174 use is recommended. Indeed, the output will saturate at a 175 higher $V_{\text {in }}$ input voltage value (up to several hundred volts) 176 while preserving the same sensitivity. Feedback capacitor $C 177$ will integrate the current due to external force variation and 178 applied voltage (depending on $C_{R} / C_{P}$ fraction). An electro- 179 mechanical relay-switch $k$ (in series with several kilo ohms 180 resistor) allows resetting $V_{\text {out }}$ voltage from DSpace environ- 181 ment in order to avoid the saturation. Electronic switches are 182 not suitable because of their "off" source/drain leakage cur- 183 rents. Further details and propositions are discussed in 184 Sec. V.

Output voltage is

$$
V_{\text {out }}=-\frac{1}{C} \int_{0}^{T} i(t) d t=-\frac{1}{C} Q,
$$

where, for the free beam $\left(F_{\text {ext }}=0\right)$, charge is

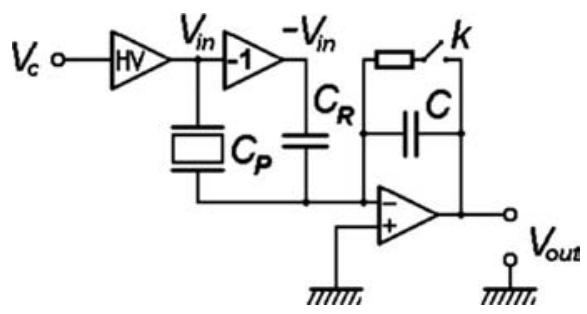

FIG. 6. Electronic circuit schematic of charge amplifier. 


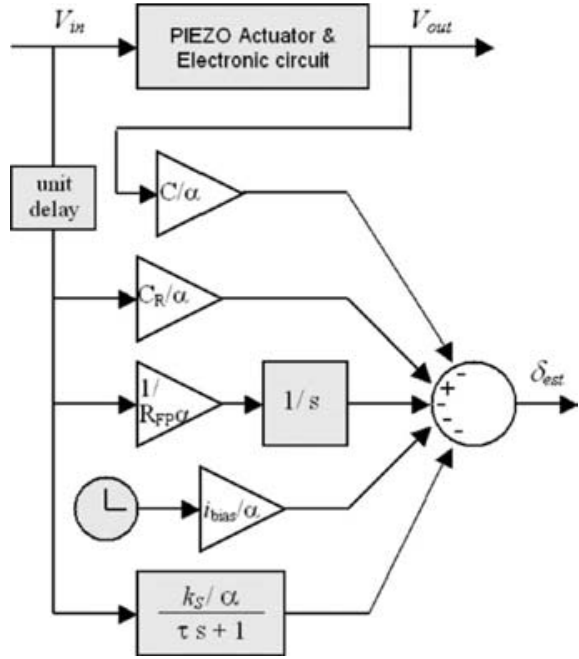

FIG. 7. Displacement detection model (Simulink).

189

$$
Q=-C_{R} V_{\text {in }}+\alpha \delta,
$$

190 where $\alpha$ was introduced in Eq. (3).

191 If we consider a nonlinear dielectric absorption effect of 192 piezoelectric material, we propose the following slight modi193 fication:

$194 Q=-C_{R} V_{\text {in }}+\left(\alpha \delta+Q_{\mathrm{DA}}\right)$,

195 where $Q_{\mathrm{DA}}$ is an internal amount of charge depending on $\varepsilon_{33}$ 196 variation.

\section{D. Detected displacement formula}

198 Adding the influence of the nonzero bias current $i_{\text {BIAS }}$ of 199 the operational amplifier (op-amp) and finite leaking resis200 tance $R_{\mathrm{FP}}$ of the piezoactuator, output voltage $V_{\text {out }}$ of the free 201 cantilever beam is given by

$$
\begin{aligned}
V_{\mathrm{out}}= & \frac{C_{R}}{C} V_{\mathrm{in}}-\frac{\alpha \delta+Q_{\mathrm{DA}}}{C}-\frac{1}{C} \int \frac{V_{\mathrm{in}}(t)}{R_{\mathrm{FP}}} d t \\
& -\frac{1}{C} \int i_{\mathrm{BIAS}}(t) d t .
\end{aligned}
$$

204 Extracting the displacement $\delta$, we obtain the estimate as 205 follows:

206

$$
\delta_{\mathrm{est}}=-\frac{C}{\alpha} V_{\mathrm{out}}-\frac{Q_{\mathrm{DA}}\left(V_{\mathrm{in}}, t\right)}{\alpha}+\frac{C_{R}}{\alpha} V_{\mathrm{in}}--\frac{1}{R_{\mathrm{FP}} \alpha} \int V_{\mathrm{in}}(t) d t
$$

$$
-\frac{1}{\alpha} \int i_{\mathrm{BIAS}}(t) d t
$$

208 We will consider a simple relaxation effect described by a 209 first-order transfer function for the dielectric absorption term

210

$$
Q_{\mathrm{DA}}^{*}(s)=\frac{Q_{\mathrm{DA}}(s)}{\alpha}=\frac{k_{s}^{*}}{\tau s+1},
$$

211 where static gain $k_{s}^{*}=k_{s} / \alpha$. Based on the previous equations, 212 Fig. 7 presents the detailed estimation bloc-scheme. Some 213 parameters of the identification in Eq. (8) have to be identi214 fied. It will be presented in the next section.

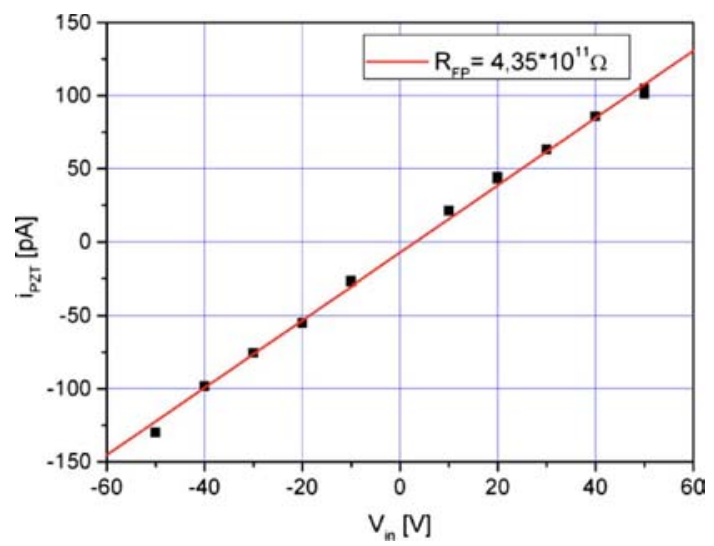

FIG. 8. (Color online) Leakage current of PZT actuator measured under constant dc voltage values. Calculated insulation resistance is $0.435 \mathrm{~T} \Omega$.

\section{SELF-SENSING PARAMETER IDENTIFICATION}

Parameters identification of Eq. (8) can be performed 216 under a manual or semiautomatic procedure. Capacitances 217 are given $\left(C=47 \mathrm{nF}\right.$ and $C_{R}=8.2 \mathrm{nF}$ in our case). The 218 identification procedure for the rest of parameters 219 ( $\alpha, i_{\mathrm{BIAS}}, R_{\mathrm{FP}}$, and $\left.Q_{\mathrm{DA}}\right)$ is based on Eq. (7), where the 220 displacement $\delta$ is provided by the displacement sensor (op- 221 tical or interferometer). The following steps describe the 222 identification procedure.

\section{A. Bias current $i_{\mathrm{BIAS}}$ identification}

Under $F_{\text {ext }}=0, V_{\text {in }}=0, V_{\text {out }} \cong 0$, and zero temperature 225 change, there is no electric current through the piezoelectric 226 material; the $V_{\text {out }}$ rate of change is measured for several doz- 227 ens of seconds, deriving $i_{\text {BIAS }}$.

228

\section{B. Leaking resistance $\boldsymbol{R}_{\mathrm{FP}}$ identification}

Under $F_{\text {ext }}=0$, a constant voltage $V_{\text {in }} \neq 0$ is applied to the 230 actuator. After several hundred seconds the creep influence 231 becomes negligible, and the output voltage $V_{\text {out }}$ shifts with a 232 constant slope, depending on $i_{\mathrm{BIAS}}$ (identified before) and $R_{\mathrm{FP}} 233$ (to be identified).

234

The identification can be repeated for different $V_{\text {in }}$ values 235 and averaged. Each point in Fig. 8 was recorded after a 236 1000-2000 s delay, to eliminate residual creep influence. 237 Linear regression was applied.

Quality piezocantilevers will exhibit $R_{\mathrm{FP}}$ values superior 239 to $1010 \Omega$. For our actuator we identified $R_{\mathrm{FP}}=0.435 \mathrm{~T} \Omega . \quad 240$

\section{Displacement coefficient $\alpha$ identification}

A step signal is applied on the free actuator. To avoid 242 dynamic oscillations of the actuator, the step signal is shaped 243 with ramp of around $20 \mathrm{~V} / \mathrm{s}$ (Fig. 9). Measured values of $\delta 244$ and $V_{\text {out }}$ immediately after $V_{\text {in }}$ step signal will serve to com- 245 pute $\alpha$

$$
\alpha=\left(-C V_{\text {out }}+C_{R} V_{\text {in }}\right) / \delta .
$$

An alternate method for deriving $\alpha$ is to apply one or several 248 sinusoidal signals as in Fig. 10 and use amplitude values in 249 Eq. (10). 

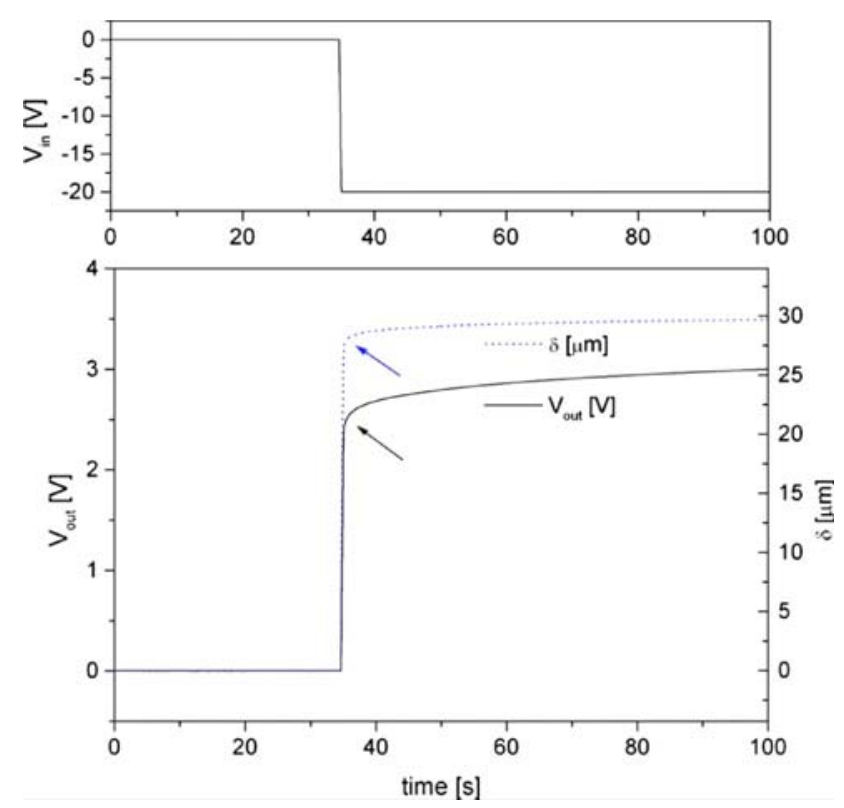

FIG. 9. (Color online) Identification of $\alpha$ coefficient with a $-20 \mathrm{~V}$ ramped step input.

\section{D. Identification of dielectric absorption transfer 252 function}

253 The last part to be identified in displacement in Eq. (8) is 254 the dielectric absorption $Q_{\mathrm{DA}}\left(V_{\mathrm{in}}, t\right)$ of the piezoelectric 255 material.

256

$$
\Delta \delta_{\text {est }}(s)=Q_{\mathrm{DA}}^{*}(s) V_{\text {in }}(s),
$$

257 where $\Delta \delta_{\text {est }}=\delta_{\text {est }}-\delta$ is the difference between estimated (us258 ing already identified parameters) and measured tip displace259 ments (Fig. 11). Identification of $k_{s}$ and $\tau$ is performed on a 260 step response, calculating the static gain and response time to 261 reach $63.2 \%$ of final value.
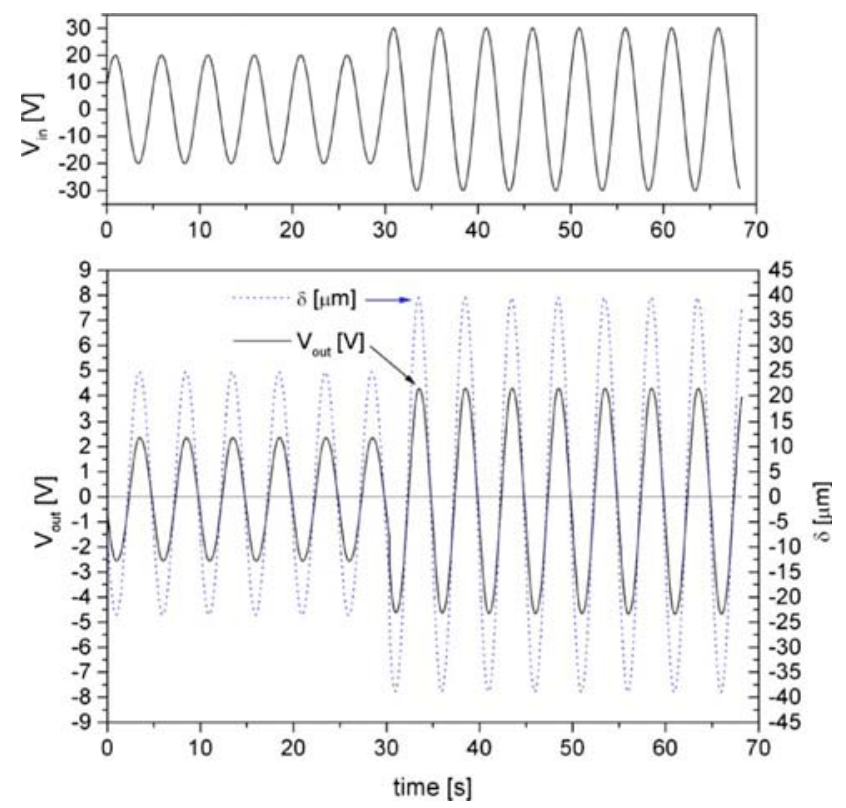

FIG. 10. (Color online) Identification of $\alpha$ coefficient from sinusoidal signals.
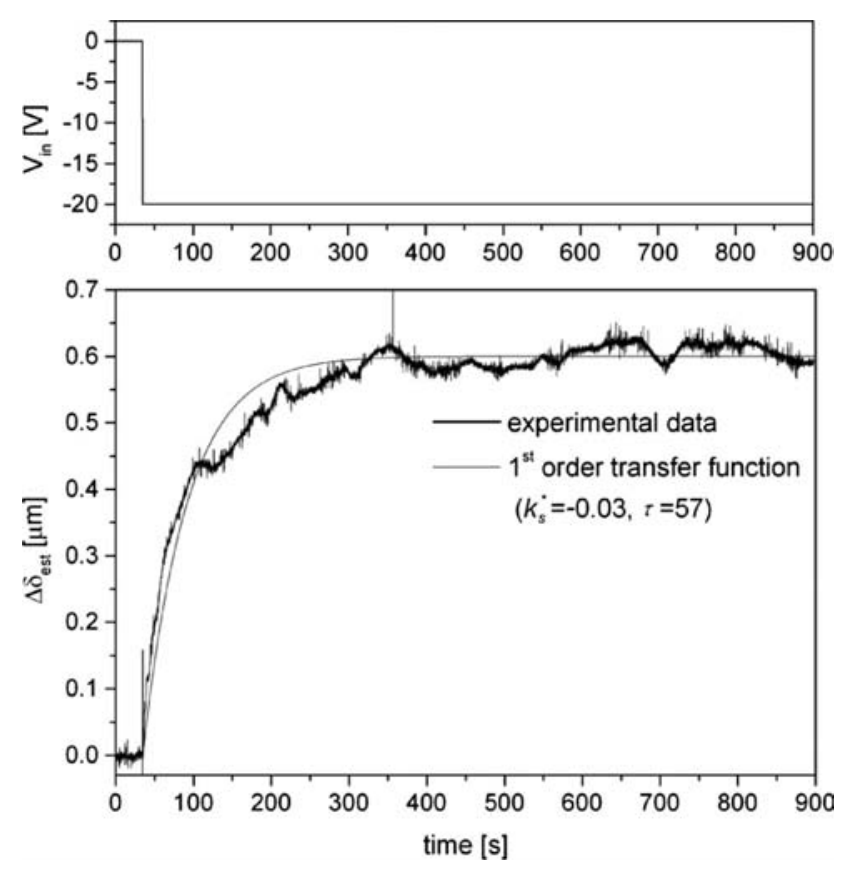

FIG. 11. Identification of dielectric absorption $Q_{\mathrm{DA}}^{*}(s)$ transfer function.

\section{SELF-SENSING RESULTS}

Several tests have been performed to evaluate the accu- 263 racy of the proposed self-sensing technique. Known and 264 identified parameters are entered into the real-time processor, 265 we have

266

$$
\begin{array}{ll}
\alpha=-10.05 e^{-9} \mathrm{C} / \mathrm{m}, & 267 \\
C=47 e^{-9} \mathrm{~F}, & 268 \\
C_{P}=1.74 e^{-9} \mathrm{~F}, & 269 \\
C_{R}=8.2 e^{-9} \mathrm{~F}, & 271 \\
R_{\mathrm{FP}}=0.435 e^{12} \Omega, & 272 \\
i_{\text {BIAS }}=-1.7 e^{-12} \mathrm{~A}, & 273 \\
\tau=57 \mathrm{~s}, & 274 \\
k_{s}=3.02 e^{8} \mathrm{~m} / \mathrm{V} . &
\end{array}
$$

\section{A. Displacement self-sensing results}

275

In Fig. 12, an input signal $V_{\text {in }}$ was applied in several 276 steps between +20 and $-25 \mathrm{~V}$, under null external force. 277 Data was recorded for 1020 s-largely sufficient for most 278 applications involving piezoelectric actuators. A very good 279 agreement is found; measured and detected displacement 280 curves almost superpose.

281

A comparative representation of displacement errors is $\mathbf{2 8 2}$ made as follows. Three graphs are traced (Figs. 13-15) from 283 uncompensated to fully compensated with respect to leaking 284 resistance and dielectric absorption. Measurement with Key- 285 ence optical displacement reader provided a poorer linearity 286 than self-sensing signal, making it impossible for accurate $\mathbf{2 8 7}$ error evaluation; SIOS interferometer was eventually em- 288 

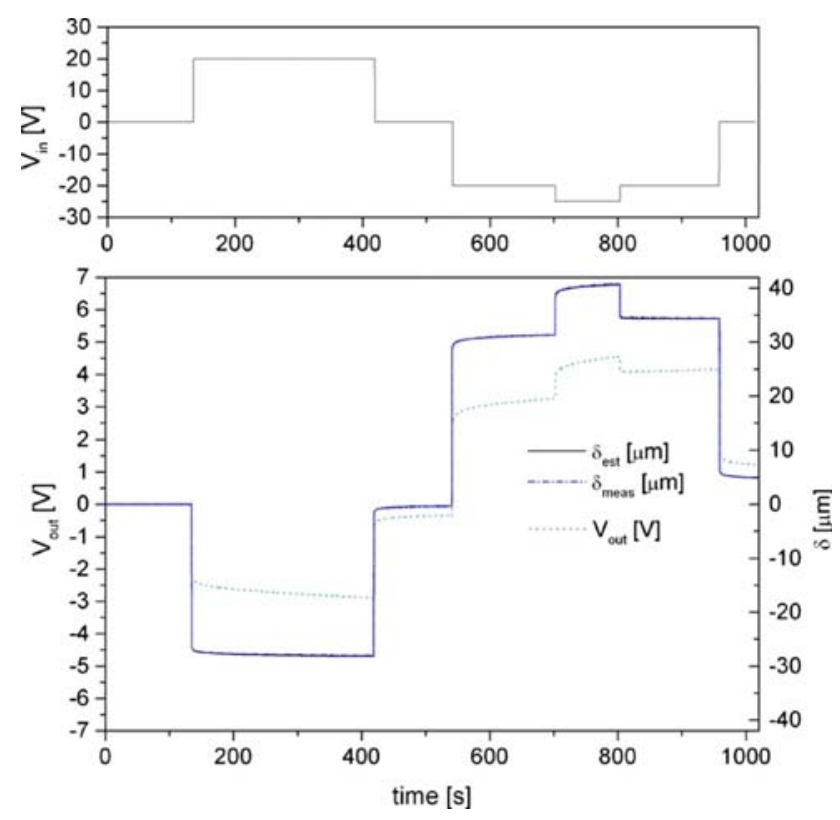

FIG. 12. (Color online) Measured and detected displacement for an arbitrary $V_{\text {in }}$ input signal $\left(F_{\text {ext }}=0\right)$.

289 ployed. Our constraint on the utilized interferometer is that 290 data is only available offline. Vertical error lines in the fig291 ures can be neglected and are due to the linear interpolation 292 and sampling period mismatch between the two data sets 293 (acquired at sampling rates of 10 and $16.11 \mathrm{~Hz}$ ).

294 As seen in the Fig. 13, peak-to-peak error of uncompen295 sated signal is $2.75 \mu \mathrm{m}$. Compensation of $R_{\mathrm{FP}}$ leaking resis296 tance allowed a reduction in maximum error to $1.05 \mu \mathrm{m}$ 297 (Fig. 14). Adding the compensation of dielectric absorption 298 (Fig. 15) provided a $0.38 \mu \mathrm{m}(0.55 \%)$ peak-to-peak error. 299 Unaveraged measured self-sensing signal noise in dis300 placement is of only $1.6 \mathrm{~nm}$ ( $\mathrm{rms}$ ), being 10 times less noisy 301 than that of filtered Keyence LC-2420 sensor (16.7 nm rms 302 noise on 4096 averaged samples). However, as expected, 303 SIOS SP 120 interferometer showed best results: $0.5 \mathrm{~nm} \mathrm{rms}$ 304 noise (Fig. 16).

\section{B. Temperature influence on displacement self- 306 sensing accuracy}

307 Temperature exhibits changes in dielectric and piezo308 electric constants. Also, differences in thermal expansion of

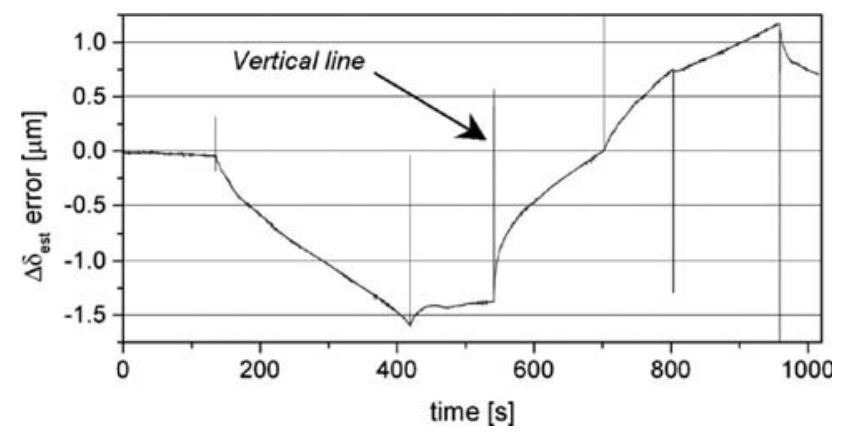

FIG. 13. Error curve of detected displacement with no leaking resistance $R_{\mathrm{FP}}$ and dielectric absorption $Q_{\mathrm{DA}}$ compensation.

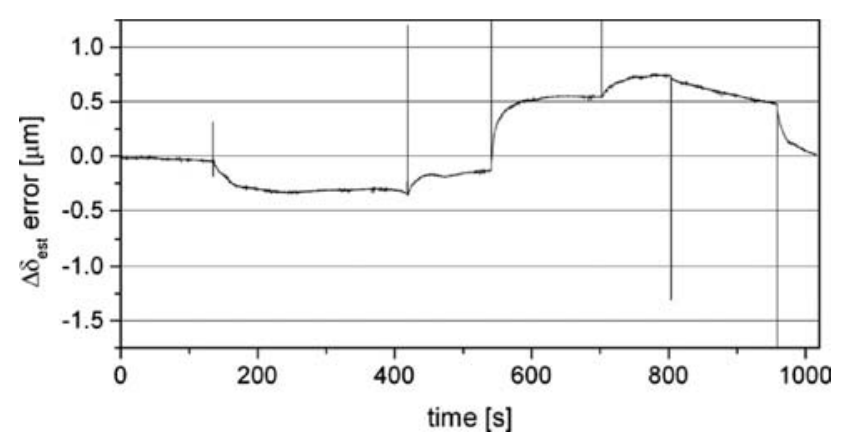

FIG. 14. Error curve of detected displacement with only leaking resistance $R_{\mathrm{FP}}$ compensation.

piezoelectric and passive material tend to bend the structure 309 like a thermal bimetallic, conducting to parasitic displace- 310 ment (and charges). In this case Eq. (2) between charge and 311 displacement no longer applies $(Q \neq \alpha \delta)$, leading to dis- 312 placement errors. To analyze the thermal influence, we com- 313 pared its effects on two types of piezoelectric beams: uni- $\mathbf{3 1 4}$ morph and bimorph cantilevers. As seen in figures and as $\mathbf{3 1 5}$ expected, unimorphs (Fig. 17) are more affected by ambient 316 temperature than bimorphs (Fig. 18). As bimorph cantilevers 317 are intrinsically symmetric, charges from both sides sum up $\mathbf{3 1 8}$ and self-compensate.

319

If we compare the above results, we see that unimorph 320 cantilevers are five times more sensitive to temperature than 321 bimorphs. Errors can be limited by a proper thermal isolation 322 or compensated with a sensitive temperature sensor like a 323 miniature thermistor. However, temperature sensor should be $\mathbf{3 2 4}$ in contact with the actuator for more correlate readings. $\quad 325$

\section{CURRENT INTEGRATION RELATED ISSUES}

326

An improper choice of charge amplifier ${ }^{21}$ will signifi- 327 cantly reduce sensing accuracy. The circuit should be pro- 328 tected against temperature changes, with a special care to 329 PCB design (guard rings, sufficient space between routes, 330 vias, and pads) otherwise unwanted leakage will easily ex- 331 ceed op-amp bias current.

Integrating capacitor must have primarily an extremely 333 high insulation resistance, low dissipation factor, and good 334 temperature stability. Polypropylene plastic film capacitors 335 were employed in our case, with a measured leaking resis- 336 tance of $24 \mathrm{~T} \Omega$ for $C=10 \mathrm{nF}$, high enough to ignore its 337

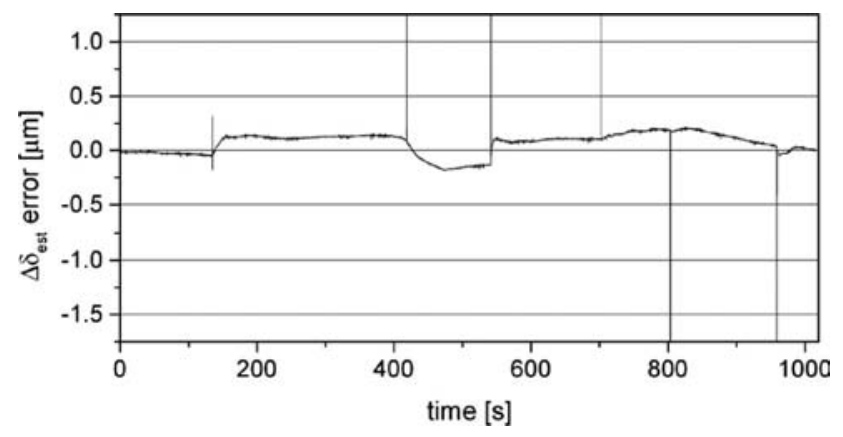

FIG. 15. Error curve of detected displacement with compensation of leaking resistance $R_{\mathrm{FP}}$ and dielectric absorption $Q_{\mathrm{DA}}$. 


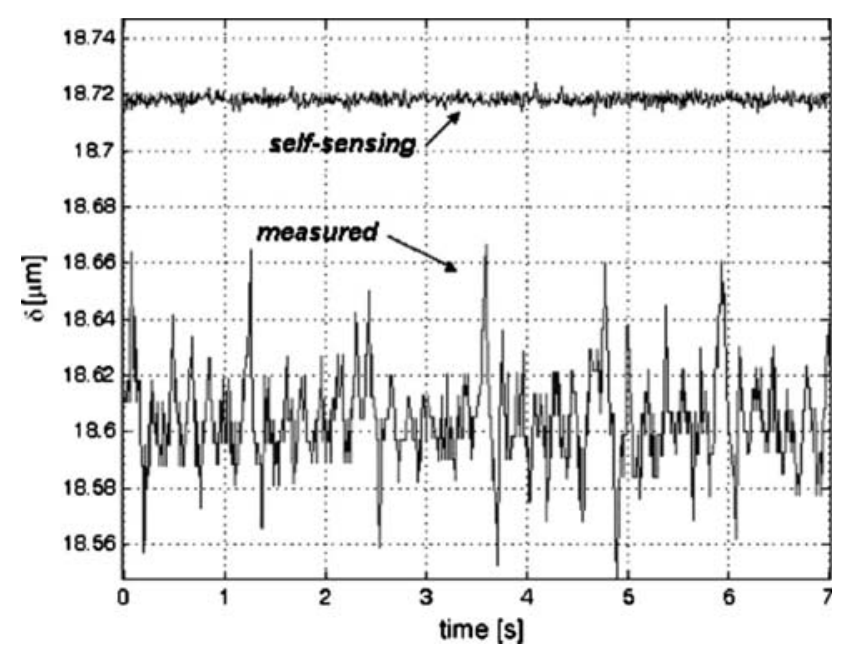

FIG. 16. Zoomed in measure (Keyence LC2420) and detected displacement for noise evaluation.

338 leaking influence in the circuit. Polystyrene or Teflon capaci339 tors also showed better performance than ceramic or polyes340 ter film capacitors.

341 Precise operational amplifiers used in charge amplifiers 342 must be unity-gain stable; otherwise they will tend to oscil343 late. Noise and bias currents have to be as small as possible. 344 Several op-amp types were tested, OPA111BM Difet model 345 was chosen for its very small bias current (1.7 pA), small 346 offset voltage, small temperature drift, fair supply voltage, 347 and on-chip guarding ring. OPA627 model is also suitable. $348 \quad$ Attention has must be paid to supply and input voltages. 349 The circuit is damaged if high input voltage is applied in the 350 absence of supply voltage. Also, to prevent the output satu351 ration, the $k$ switch allows resetting when necessary. Further 352 increase in voltage over an already saturated op-amp will 353 cause damage.

354 Cables should be shielded properly to avoid the electro355 magnetic interference. Further noise rejection can be 356 achieved by modifying the electronic schematic presented in
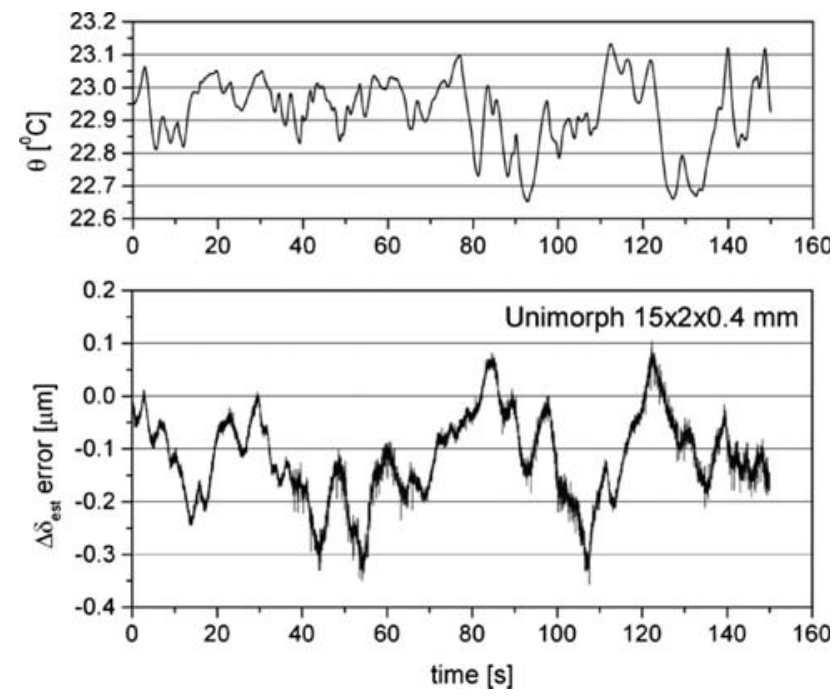

FIG. 17. Typical self-sensing displacement error due to ambient temperature change in a unimorph actuator. Error is $\sim 1 \mu \mathrm{m} /{ }^{\circ} \mathrm{C}$.
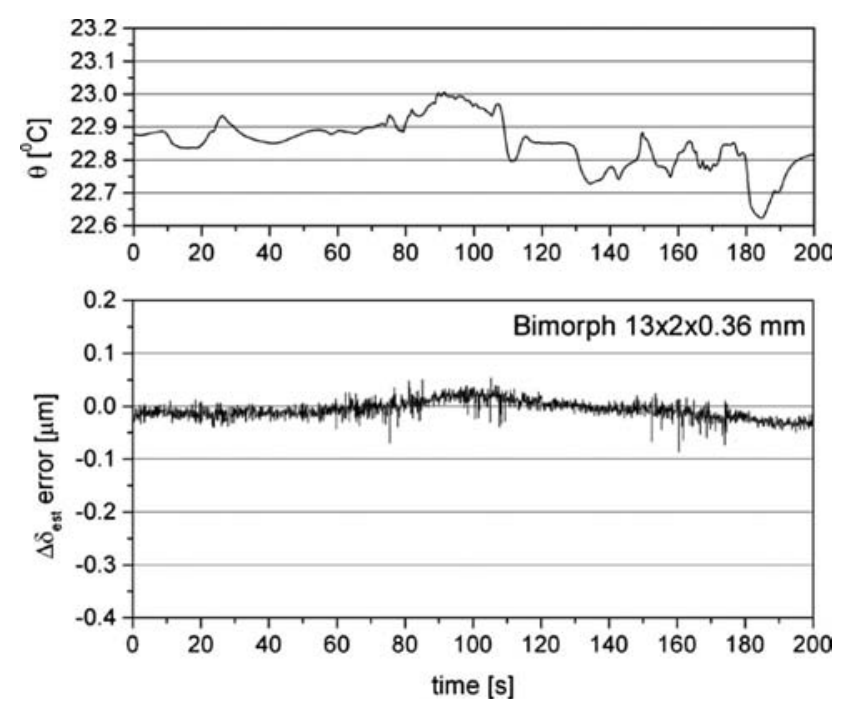

FIG. 18. Typical self-sensing displacement error due to ambient temperature change in a bimorph actuator. Error is $\sim 0.2 \mu \mathrm{m} /{ }^{\circ} \mathrm{C}$.

Fig. 6. We propose the schematic pictured in Fig. 19 where 357 the current, proportional to the voltage drop across a series 358 resistance or more likely across a voltage divider (to avoid 359 op-amp damage due to HV), is buffered or preamplified and $\mathbf{3 6 0}$ then integrated. Indeed, this schematic allows noise reduc- 361 tion thanks to the grounded series resistance $R_{Z_{2}}$ connected to 362 the high impedance amplifier input.

$$
V_{\text {out }}=-\frac{R_{Z_{2}}}{R C\left(R_{Z_{1}}+R_{Z_{2}}\right)} \int_{0}^{T} i(t) d t,
$$

For our actuator the best compromise between response time, 365 sensitivity, and noise was a series resistance of $82 \mathrm{k} \Omega\left(R_{Z_{1}} 366\right.$ $+R_{Z_{2}}=82 \mathrm{k} \Omega$ ).

367

Noise was reduced by a factor of five but on the other 368 hand this schematic was much more sensitive to temperature 369 offset drifts than that of Fig. 6. As $V_{z}$ voltage is in the $\mu \mathrm{V} 370$ range or lower, op-amp offset voltage temperature drift 371 $\left( \pm 0.5 \mu \mathrm{V} /{ }^{\circ} \mathrm{C}\right)$ and supply rejection $( \pm 3 \mu \mathrm{V} / \mathrm{V})$ limited 372 system accuracy. Usually op-amp offset is trimmed manually 373 (with potentiometers); in our case this measure was not suf- $\mathbf{3 7 4}$ ficient to compensate thermal drifts. We made an automatic 375 compensation of the offset voltage with random temperature 376 changes. This was performed by connecting DSpace DAC 377 outputs (Fig. 20) to op-amp "trim" pins and by measuring 378 and referencing the temperature to a miniature thermistor in $\mathbf{3 7 9}$ close contact with op-amp chip. This way, we preserved a $\mathbf{3 8 0}$ signal up to $100 \mathrm{~s}$ similar in accuracy with that of Figs. 381 $12-15$, however rms noise was reduced from $1.6 \mathrm{~nm}$ to only 382 $0.4 \mathrm{~nm}$, inferior to even that of SIOS SP120 interferometer. 383

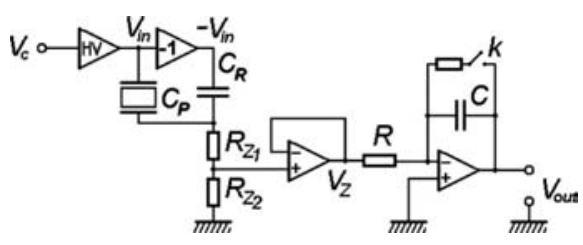

FIG. 19. Alternate schematic with voltage divider and integrator allowed noise reduction. 


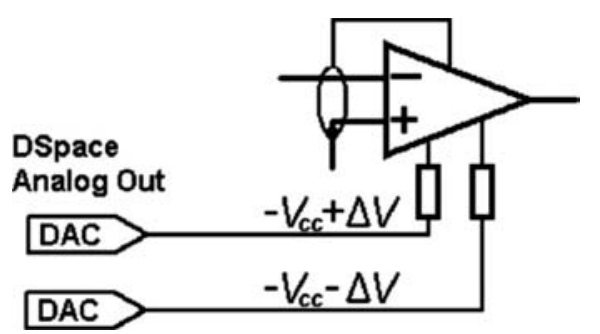

FIG. 20. Offset drift compensation connecting DSpace output to trim pins.

384 Zero-drift chopper op-amps (typically $\pm 0.03 \mu \mathrm{V} /{ }^{\circ} \mathrm{C}$ ) will 385 probably ameliorate temperature drifts but other effects such 386 as thermal electro motive force (EMF) (Seebeck effect) in 387 cable junctions will still perturbate the circuit.

388 To generally resume, charge integration is prone to non389 zero bias currents offset voltages, temperature drifts, leaking 390 resistance or currents, thermal EMF and electro magnetic 391 interference influence. However, with proper measures, their 392 influence can be eliminated or at least partially quantified 393 and compensated.

\section{VI. CONCLUSION}

395 Displacement self-sensing of uni- and bimorph cantile396 vered actuators used for meso- and microscale gripping and 397 manipulation is cost-effective and relatively simple to imple398 ment or upgrade to existing systems. To our knowledge it is 399 a first paper focusing on displacement self-sensing of these 400 devices. We referred to a current integration method self401 compensated against some actuator nonlinearities (hysteresis 402 and creep) and externally compensated to others (leaking re403 sistivity and dielectric absorption).

404 In the case of detected or a priori-supposed absence of 405 external forces, displacement is almost directly proportional 406 to the charge. Further compensation of nonzero amplifier 407 bias current, finite actuator leaking resistance, and dielectric 408 absorption lead to a significant reduction in errors, up to $4090.55 \%$ and an increase in measurement period to more than $4101000 \mathrm{~s}$, sufficient enough for most tasks. Signal noise was 411 lower than that measured with expensive laser triangulation 412 sensor. Two schematics were presented, the first one based 413 on direct current integration showed its feasibility for long 414 integration periods while the second integrating shunt volt415 age drop allowed a further reduction in signal noise with a cost of a more unstable long-term signal. Practical issues $\mathbf{4 1 6}$ related to long-term charge preservation were presented, and 417 temperature influence discussed.

\section{ACKNOWLEDGMENTS}

The authors especially thank Roger Bourquin, professor 420 at ENSMM Besancon, France, for helpful discussions. This 421 work is supported by the EU FP7 SP3-People Program under 422 Grant No. PIEF-GA-2008-219412 (New Micro-Robotic 423 Systems featuring Piezoelectric Adaptive MicroStructures 424 for Sensing and Actuating, with Associated Embedded 425 Control).

${ }^{1}$ T. S. Low and W. Guo, J. Microelectromech. Syst. 4, 230 (1995). 427

${ }^{2}$ H. J. M. T. A. Adriaens, W. L. de Koning, and R. Banning, IEEE/ASME 428 Trans. Mechatron. 5, 31 (2008). 429

${ }^{3}$ Y.-G. Zhou, Y.-M. Chen, H.-J. Ding, and W.-Q. Chen, J. Zhejiang Univ. 430 Sci. A. 9, 1 (2008).

${ }^{4}$ R. J. Wood, E. Steltz, and R. S. Fearing, Proceedings of the IEEE Inter- 432 national Conference on Robotics and Automation, Spain, 2005 (unpub- 433 lished).

${ }^{5}$ H. Jung and D.-G. Gweon, Rev. Sci. Instrum. 71, 1896 (2000). 435

${ }^{6}$ M. Rakotondrabe, C. Clévy, and P. Lutz, IEEE Trans. Autom. Sci. Eng. 436 (unpublished). 437

${ }^{7}$ M. Rakotondrabe, Y. Haddab, and P. Lutz, IEEE Trans. Control Syst. 438 Technol. 17, 528 (2009). 439

${ }^{8}$ M. Rakotondrabe, Y. Haddab, and P. Lutz, Proceedings of the IEEE/ 440 ASME International Conference on Advanced Intelligent Mechatronics, 441 Zurich, Switzerland, September 2007 (unpublished), pp. 1-6. 442

${ }^{9}$ J. J. Dosch, D. J. Inman, and E. Garcia, J. Intell. Mater. Syst. Struct. 3, 166443 (1992).

${ }^{10}$ W. W. Law, W.-H. Liao, and J. Huang, Smart Mater. Struct. 12, 720445 (2003).

${ }^{11}$ P. C. Khiang, G. Guoxiao, M. B. Chen, and T. H. Lee, IEEE/ASME Trans. 447 Mechatron. 11, 328 (2006).

${ }^{12}$ G. A. Lesieutre, Shock Vib. Dig. 30, 187 (1998).

S. O. R. Moheimani, IEEE Trans. Control Sys. Technol 11, 482 (2003). 450

${ }^{14}$ S. O. R. Moheimani and Y. K. Yong, Rev. Sci. Instrum. 79, 073702451 (2008).

${ }^{15}$ S. O. R. Moheimani, Rev. Sci. Instrum. 79, 071101 (2008). 453

${ }^{16}$ D. Campolo, R. Sahai, and R. S. Fearing, Proceedings of the IEEE Inter- 454 national Conference on Robotics and Automation, Taipei, 2003 (unpub- 455 lished), Vol. 3, pp. 3339-3346.

${ }^{17}$ Y. Cui, Proceeding of the Sixth World Conference on Intelligent Control 457 and Automation, China, 2006 (unpublished). 458

${ }^{18}$ J. Agnus and N. Chaillet, INPI Patent No. FR 03000532 (August 2004). 459

${ }^{19}$ A. J. Fleming and S. O. R. Moheimani, Rev. Sci. Instrum. 76, 073707460 (2005).

${ }^{20}$ J. G. Smits and W.-S. Choi, IEEE Trans. Ultrason. Ferroelectr. Freq. 462 Control 38, 256 (1991).

${ }^{21}$ J. Karki, Texas Instr. Appl. Report No. SLOA033A, 2000.

7

8

10

33

435

(3)

437

438

0

44

